\title{
Heavy-flavour production processes relevant for PDF fits
}

\section{Sergey Alekhin}

II Institut für Theoretische Physik, Universität Hamburg,

E-mail: sergey.alekhin@desy.de

\section{Michael Benzke}

II Institut für Theoretische Physik, Universität Hamburg,

E-mail: michael.benzke@desy.de

\section{Giuseppe Bevilacqua}

MTA-DE Particle Physics Research Group, Hungarian Academy of Sciences and University of Debrecen,

E-mail: giuseppe.bevilacqua@science.unideb.hu

\section{Maria Vittoria Garzelli*}

Dipartimento di Fisica e Astronomia, Università degli Studi di Firenze, \& INFN, Firenze, Institut für Theoretische Physik, Eberhard Karls Universität Tübingen,

E-mail: maria.vittoria.garzelliddesy.de

\section{Adam Kardos}

Institute of Physics, University of Debrecen,

E-mail: adam.kardosescience.unideb.hu

\section{Bernd Kniehl}

II Institut für Theoretische Physik, Universität Hamburg,

E-mail: bernd.kniehl@desy.de

\section{Sven-Olaf Moch}

II Institut für Theoretische Physik, Universität Hamburg,

E-mail: sven-olaf.moch@desy.de

\section{Oleksandr Zenaiev}

II Institut für Theoretische Physik, Universität Hamburg,

E-mail: oleksandr.zenaiev@desy.de

We present recent developments in the theoretical description of processes leading to the production of heavy flavours, which, together with the most recent experimental data, are of interest for further constraining fits of Parton Distribution Functions. We compare different approaches, and, for selected cases, show the impact of using the $\overline{\mathrm{MS}}$ scheme as an alternative to the on-shell scheme for renormalizing the heavy-quark masses.

XXVII International Workshop on Deep-Inelastic Scattering and Related Subjects - DIS2019 8-12 April, 2019

Torino, Italy

\footnotetext{
* Speaker.
} 


\section{Introduction}

Heavy-flavour production processes allow to constrain different aspects of Parton Distribution Functions (PDF) of protons and nuclei.

In ep semi-inclusive Deep Inelastic Scattering (DIS), charm (bottom) production can be used to constrain the charm (bottom) quark mass values, which are fitted together with the dependence of the gluon PDF on the longitudinal momentum fraction $x$, considering the correlations between these quantities [1]. In fact these mass values are an input for the theory predictions of inclusive DIS that are compared to experimental data at the core of all PDF fit procedures. Inclusive DIS data allow to indirectly constrain the $x$ dependence of the gluon distribution, in the interval $10^{-3} \lesssim x \lesssim 10^{-1}$ for the kinematics of the HERA collider. Additionally, the data on charm and bottom production in semi-inclusive DIS also have some direct impact on these distributions and on the strange sea ones. At HERA, the gluon $x$ values probed by charm (bottom) production in semi-inclusive DIS cover the range $10^{-4} \lesssim x \lesssim 0.05(0.002<x<0.04)$.

Inclusive $D$-meson production in $p p$ collisions at the Large Hadron Collider (LHC) has been used to constrain the gluon and sea quark distributions at low $x$ values [2,3]. In particular, the larger the collider center-of-mass energy $\sqrt{s}$, the lower the $x$ values probed. For $\sqrt{s}=13 \mathrm{TeV}$, the constraints extend down to $x \gtrsim 10^{-6}$. These investigations have been extended to the case of $p A$ collisions in Ref. [4]. On the other hand, $B$-meson production measured by LHCb can be used to constrain the gluon and sea quark distributions at very large $x(x>0.1)$ [2], and top-antitop quark production measured by CMS is also sensitive to gluon PDFs at large $x$ 's, $0.001 \lesssim x \lesssim 0.5$ [5] (see also Ref. [6]). Finally, the production of a charm quark in association with a $W$ boson allows to constrain strange sea PDFs at intermediate $x$ 's, whereas single top production in the $t$-channel and in the $W$ - top associated production channel, allow to constrain bottom quark PDFs. In the following, we focus on two of these processes, $W+$ charm and inclusive $D$-meson production, and show some recent developments and still open issues in their theoretical description, that can be of interest for more precise PDF fits.

\section{Charm production in association with a $W$ boson}

When considered at leading order in the $\alpha_{s}$ expansion, and in absence of mixing between quarks of different families, $W$ production in association with a single charm quark in $p p$ collisions can be regarded as a direct probe of the strange quark distribution, considering that the only possible QCD hard-scattering processes are $g \bar{s} \rightarrow W^{+} \bar{c}$ and $g s \rightarrow W^{-} c$. The sensitivity to strange quark PDFs is actually weakened by the consideration that $V_{\mathrm{CKM}}$ is non-diagonal, and by the fact that radiative corrections have to be taken into account. These two elements open up $W+$ charm production channels where quarks different from the strange one can contribute. In particular the $d$ quark contributes already at leading order. Additionally, at NLO, even the $g g$ channel contributes. Recently, we have extended the PowHel event generator, to the computation of $W+$ charm production. PowHel makes use of tree-level and one-loop matrix-elements provided by the Helac-NLO [7] set of codes and of the algorithms in POWHEGBOX [8] to produce predictions at next-to-leading order (NLO) and events at the first radiation emission level, according to the POWHEG [9] NLO + Parton Shower (PS) matching framework. These events, stored 
in files in the Les Houches (LHE) format, are ready to be further showered by Shower Monte Carlo (SMC) event generators (like e.g. PYTHIA) [10], to produce predictions including parton shower, hadronization and further effects (multiple parton interactions, beam remnant effects, etc.), which can be directly compared with experimental data at particle level. Charm mass effects are included throughout our computations, with hard-scattering matrix-elements and PDFs in the 3 flavour-number scheme, and charm mass values $m_{c}$ in the SMC event generator chosen consistently with the hard-scattering matrix-elements. Although a non-diagonal $V_{\mathrm{CKM}}$ can be activated in our simulations, in the following a diagonal mixing matrix is used for simplicity. $W$ leptonic decays are handled by PYTHIA. We compare POwHel + PYTHIA predictions, obtained using as input $m_{c}=1.5 \mathrm{GeV}, m_{W}=80.379 \mathrm{GeV}, \Gamma_{W}=2.085 \mathrm{GeV}, \mu_{R}=\mu_{F}=H_{T} / 2$, with $H_{T}$ defined as the sum of the transverse energies of the emitted particles at the LHEF level, and different PDF sets, to the experimental data at $\sqrt{s}=7 \mathrm{TeV}$ reported by the ATLAS collaboration [11]. These data refer to two analyses of $W+$ charm production, with the $W$ boson decaying in the $W \rightarrow \ell v$ channel, with the $p_{T, \ell}>20 \mathrm{GeV},\left|\eta_{\ell}\right|<2.5, p_{T, v}>40 \mathrm{GeV}$ and $m_{W}>40 \mathrm{GeV}$ selection cuts applied. In the first analysis, the presence of an additional $D$ or $D^{*}$ meson is required, with $p_{T, D^{(*)}}>8 \mathrm{GeV}$ and $\left|\eta_{D^{(*)}}\right|<2.2$, whereas in the second analysis the presence of exactly one $c$-jet with $p_{T, j}>25$ $\mathrm{GeV},\left|\eta_{j}\right|<2.5$, which includes at least a charmed hadron with $p_{T}>5 \mathrm{GeV}$, is required. The experiment distinguishes opposite-sign (OS) events, i.e. those where the $D$-meson (or $c$-jet) originates from a $c$-quark with charge opposite to that of the lepton emitted from the $W$, from same-sign (SS) events, where the $D$-meson (or $c$-jet) and the lepton have the same sign. The cross-sections provided refer to the difference OS-SS. This way, the contribution of the $W c \bar{c}$ background is very much reduced, considering that it approximately contributes to the same amount of OS and SS events. Fig. 1 refers to the comparison of our theoretical predictions for $\left|\eta_{\ell}\right|$ with the experimental data of the $D^{(*)}$-meson analysis, whereas Fig. 2 concerns the case of the $c$-jet analysis. For each figure, the two panels refer to the case of leptons with opposite charge. In all cases, central predictions with ABMP 16_3_nlo [12] PDFs turn out to be a few percent larger than those with CT14nlo_NF3 [13], and turn out to be in good agreement with experimental data at low $\left|\eta_{\ell}\right|$. Both these PDF fits include a strange quark distribution identical to the antistrange one. The fact that, for both analyses, central theoretical predictions for $W^{+}$production turns out to be in slightly better agreement with experimental data than for the $W^{-}$case, could point towards a slight enhancement of the strange quark distribution with respect to the antistrange one. However, in view of the theoretical uncertainties due to scale variation and fragmentation (not shown in our plots), amounting to $10-20 \%$, and of the experimental uncertainties of similar size, drawing such a conclusion can be regarded as very premature. Systematic comparisons with further data in further LHC analyses with better statistics, still distinguishing the cases of $W^{+}$and $W^{-}$[14], can help to confirm or falsify these observations.

\section{Inclusive $D$-meson production at colliders}

Different experiments have measured the production of various $D$-mesons at the Tevatron and the LHC. Their data allow to probe different $\left(Q^{2}, x\right)$ regions that have partial overlap with each other. The most interesting measurements for constraining PDFs are those at large rapidities (corresponding to more extreme $x$ values), uniquely provided by the $\mathrm{LHCb}$, which probe the $2<y<4.5$ 

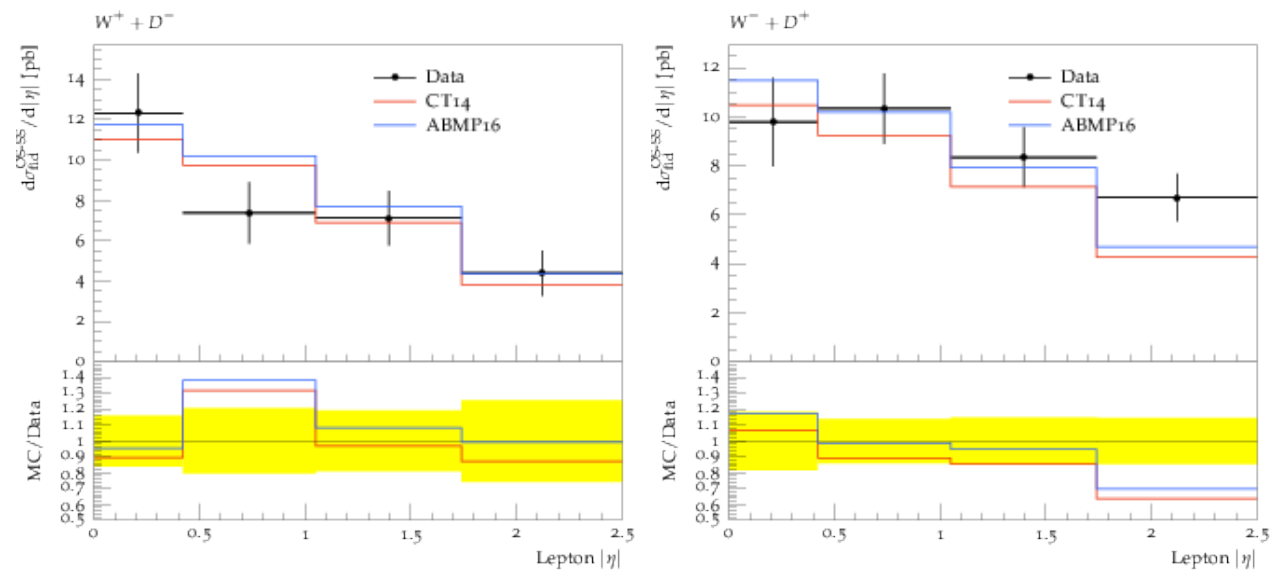

Figure 1: $|\eta|$ distribution for the lepton from $W$ decay in $W+D^{(*)}$ events, as computed by PowHel + PYTHIA [11], compared with ATLAS experimental data, obtained in the $W+D^{(*)}$ analysis. The two panels refer to leptons of opposite charge. See text for more detail.
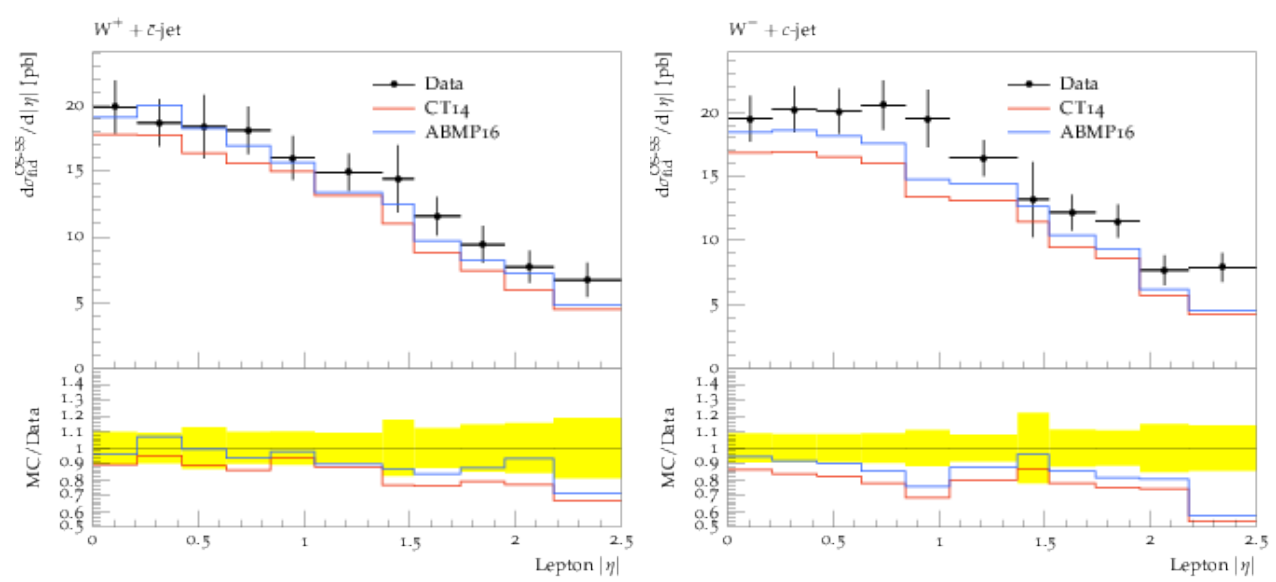

Figure 2: Same as in Fig. 1, but for the $W+c$-jet analysis. See text for more detail.

region. On the other hand, all measurements, including those at small rapidities, can help validating the various available theoretical descriptions of this process used in the QCD analyses leading to PDF fits. In the left panel of Fig. 3 we show a comparison of the experimental data for inclusive $D^{+}$ meson hadroproduction obtained by $\mathrm{CDF}$ at $\sqrt{s}=1.96 \mathrm{TeV}$ in the rapidity region $|y|<1[15,16]$, with predictions obtained by different tools. In particular we compare predictions at NLO in the fixed flavour-number scheme (FFNS) with 3 active flavours complemented by phenomenological fragmentation functions (FF), to those obtained by the POWHEGBOX+PYTHIA 8 implementation we already used in Ref. [17], that has NLO + PS accuracy, and to those obtained in the GeneralMass Variable-Flavour-Number Scheme (GM-VFNS), according to the implementation in Ref [18]. In the latter the fragmentation of partons into $D$-mesons is described by FF evolving with the factorization scale in a rigorous factorization framework. These FF were fitted in the GM-VFNS framework itself, considering data on $D$-meson production in $e^{+} e^{-}$collisions. The largest differ- 
ences among the different theoretical descriptions are visible at large transverse momentum of the produced $D$-meson $\left(p_{T}>10-15 \mathrm{GeV}\right)$. There the FFNS predictions overestimate the data, which are instead in better agreement with the GM-VFNS predictions, the only ones that resum logarithms of $p_{T} / m_{c}$ at next-to-leading logarithmic (NLL) accuracy, providing a global NLO + NLL accuracy. The predictions of POWHEGBOX+PYTHIA8 turn out to be within the GM-VFNS uncertainty bands due to scale variation, and lie close to the upper limit of these bands at large $p_{T}$. The fact that different PDFs were used (CT14nlo for the FFNS + FF and GM-VFNS predictions and ABMP 16_nlo PDF for the NLO + PS predictions), does not impact this conclusion. As shown in the right panel of Fig. 3, a similar trend is observed for the inclusive hadroproduction of $D^{+}$at the LHC at $\sqrt{s}=7 \mathrm{TeV}$, with theoretical predictions compared with experimental data obtained by the ALICE collaboration, extending on a larger range of $p_{T}$ [19].
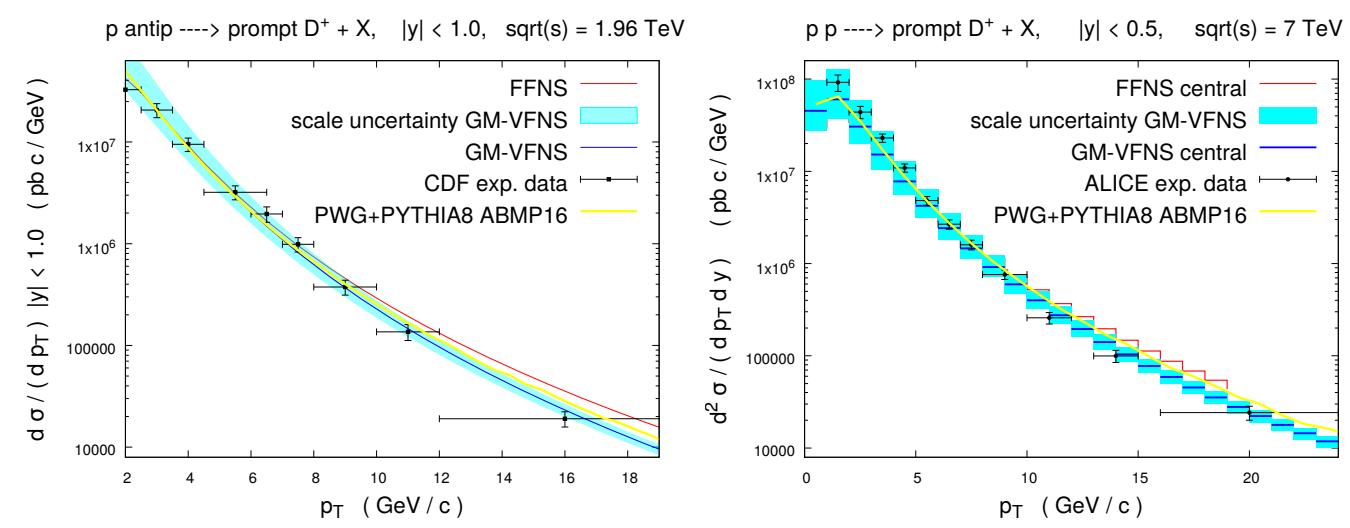

Figure 3: $\quad p_{T}$ distribution of inclusive $D^{+}$meson hadroproduction using different theoretical descriptions compared to experimental data at the Tevatron and LHC obtained by the CDF (left) and ALICE (right) collaborations, respectively. See text for more detail.

Additionally, we study the effect of changing the scheme for the renormalization of the charm quark mass on our predictions, by considering the $\overline{\mathrm{MS}}$ scheme as an alternative to the on-shell scheme. The motivation underlying this choice is the fact that the $m_{c}$ value in the $\overline{\mathrm{MS}}$ scheme [20] is plagued by a smaller uncertainty than in the on-shell scheme. In fact, it is well known that the formula for the conversion of the charm mass value from the $\overline{\mathrm{MS}}$ to the on-shell scheme, at present known exactly up to four loops [21], does not show clear evidence of convergence of the perturbative expansion. The masses in the on-shell scheme are affected by renormalon ambiguities, of the order of $\mathscr{O}\left(\Lambda_{Q C D}\right)$. The latter can severely plague the value of masses as small as the charm one, of the order $\mathscr{O}(\mathrm{GeV})$. In Fig. 4 we consider the case of inclusive $D$-meson production at low $p_{T}$ in the rapidity range $3<y<3.5$, probed by LHCb at $\sqrt{s}=7 \mathrm{TeV}$, and we show a comparison between predictions obtained in the FFNS complemented by phenomenological FF, using $m_{c}^{\overline{\mathrm{MS}}}$, and those obtained in the default implementation of the same framework, making use of $m_{c}^{\text {pole }}$, at a central scale $\mu_{R}=\mu_{F}=2 m_{c}$. Differences between central predictions in both schemes turn out to be within $10-15 \%$ and are smaller than those which arise from a change of scale, i.e. by modifying the static scale into a dynamical one $\mu_{R}=\mu_{F}=\sqrt{p_{T}^{2}+4 m_{c}^{2}}$. All these predictions are further compared to experimental data provided by the LHCb collaboration, for the $D^{+}, D^{0}, D_{s}^{+}$and $D^{*+}$ mesons [22]. 

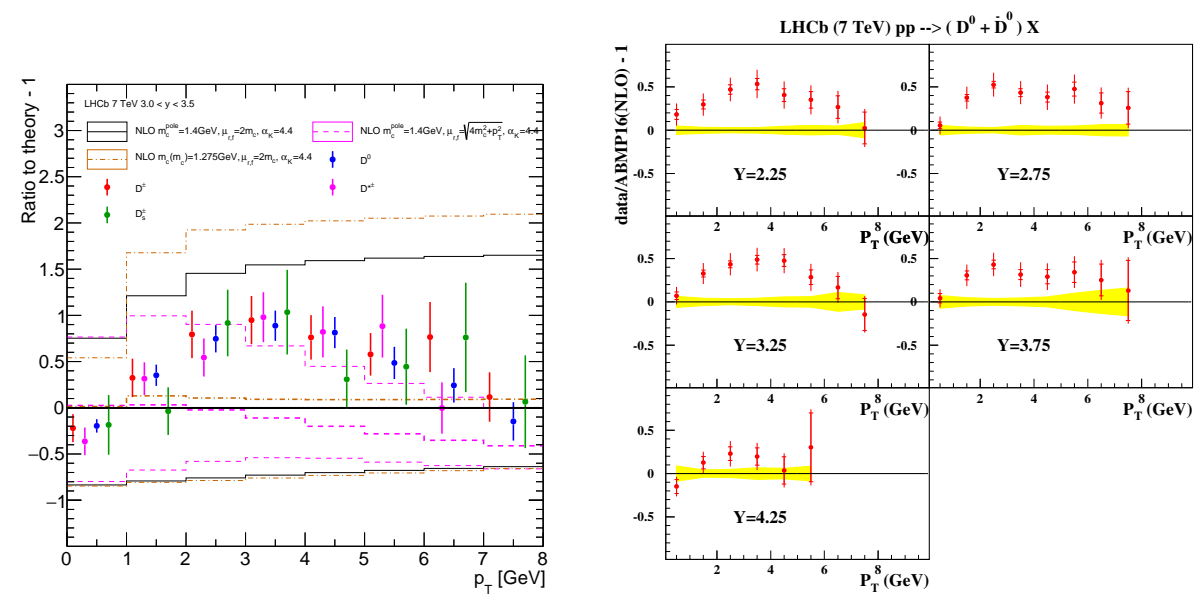

Figure 4: Ratios of experimental data for $D^{+}, D^{0}, D_{s}^{+}$and $D^{*+}$ hadroproduction obtained by LHCb at $\sqrt{s}=7 \mathrm{TeV}$ for $3<y<3.5$ and of theoretical predictions in the NLO FFNS + FF implementation with $m_{c}^{\overline{\mathrm{MS}}}$ with respect to the default implementation making use of $m_{c}^{\text {pole }}$, with $\mu_{R}=\mu_{F}=2 m_{c}$ and $n_{f}=3$. The ratio of predictions with dynamical scale $\sqrt{p_{T}^{2}+4\left(m_{c}^{\text {pole }}\right)^{2}}$, with respect to the default, is also shown. In these predictions, the ABMP 16_nlo PDF set was used. Uncertainties due to scale and charm mass variation $\left(m_{c}^{\text {pole }}=1.4 \pm 0.15 \mathrm{GeV}, m_{c}^{\overline{\mathrm{MS}}}\left(m_{c}\right)=1.27 \pm 0.03 \mathrm{GeV}\right)$ are also reported (left panel). Pulls for the LHCb $D^{0}$ data in different rapidity bins, using theory predictions from POWHEGBOX+PYTHIA (right panel).

While the data lie within the theoretical uncertainties at all $p_{T}$ values, it is clear that in the $2<p_{T}<$ $5 \mathrm{GeV}$ interval, our central predictions systematically underestimate the data by several ten percent. This observation is not influenced by the change of the charm mass renormalization scheme and points to the need of more accurate predictions, including higher-order radiative corrections, as also suggested by the fact that the theoretical uncertainties, also shown in the plot, are dominated by scale uncertainties, while other uncertainties contribute only mildly. This feature also occurs for other rapidity bins and other theory tools still using NLO hard-scattering amplitudes, as shown in Fig. 4, right.

\section{References}

[1] A. Accardi et al., A Critical Appraisal and Evaluation of Modern PDFs, Eur. Phys. J. C76 (2016), no. 8 471, [arXiv:1603.08906].

[2] PROSA Collaboration, O. Zenaiev et al., Impact of heavy-flavour production cross sections measured by the LHCb experiment on parton distribution functions at low x, Eur. Phys. J. C75 (2015), no. 8 396, [arXiv:1503.04581].

[3] V. Bertone, R. Gauld, and J. Rojo, Neutrino Telescopes as QCD Microscopes, JHEP 01 (2019) 217, [arXiv:1808.02034].

[4] A. Kusina, J.-P. Lansberg, I. Schienbein, and H.-S. Shao, Gluon Shadowing in Heavy-Flavor Production at the LHC, Phys. Rev. Lett. 121 (2018), no. 5 052004, [arXiv: 1712.07024 ].

[5] CMS Collaboration, C. Collaboration, Measurements of normalised multi-differential cross sections for top quark pair production in pp collisions at $\sqrt{s}=13 \mathrm{TeV}$ and simultaneous determination of the strong coupling strength, top quark pole mass and parton distribution functions, . 
[6] M. Czakon, N. P. Hartland, A. Mitov, E. R. Nocera, and J. Rojo, Pinning down the large-x gluon with NNLO top-quark pair differential distributions, JHEP 04 (2017) 044, [arXiv: 1611.08609 ].

[7] G. Bevilacqua, M. Czakon, M. V. Garzelli, A. van Hameren, A. Kardos, C. G. Papadopoulos, R. Pittau, and M. Worek, HELAC-NLO, Comput. Phys. Commun. 184 (2013) 986-997, [arXiv:1110.1499].

[8] S. Alioli, P. Nason, C. Oleari, and E. Re, A general framework for implementing NLO calculations in shower Monte Carlo programs: the POWHEG BOX, JHEP 1006 (2010) 043, [arXiv: 1002.2581 ].

[9] P. Nason, A New method for combining NLO QCD with shower Monte Carlo algorithms, JHEP 11 (2004) 040, [hep-ph / 0409146 ].

[10] T. Sjostrand, S. Mrenna, and P. Z. Skands, A Brief Introduction to PYTHIA 8.1, Comput. Phys. Commun. 178 (2008) 852-867, [arXiv: 0710 . 3820].

[11] ATLAS Collaboration, G. Aad et al., Measurement of the production of a $W$ boson in association with a charm quark in pp collisions at $\sqrt{s}=7$ TeV with the ATLAS detector, JHEP 05 (2014) 068, [arXiv:1402.6263].

[12] S. Alekhin, J. Blümlein, and S. Moch, NLO PDFs from the ABMP16 fit, Eur. Phys. J. C78 (2018), no. 6477 , [arXiv:1803.07537].

[13] S. Dulat, T.-J. Hou, J. Gao, M. Guzzi, J. Huston, P. Nadolsky, J. Pumplin, C. Schmidt, D. Stump, and C. P. Yuan, New parton distribution functions from a global analysis of quantum chromodynamics, Phys. Rev. D93 (2016), no. 3 033006, [arXiv: 1506.07443 ].

[14] CMS Collaboration, A. M. Sirunyan et al., Measurement of associated production of a W boson and a charm quark in proton-proton collisions at $\sqrt{s}=13$ TeV, Eur. Phys. J. C79 (2019), no. 3269 , [arXiv:1811.10021].

[15] CDF Collaboration, D. Acosta et al., Measurement of prompt charm meson production cross sections in p $\bar{p}$ collisions at $\sqrt{s}=1.96 \mathrm{TeV}$, Phys. Rev. Lett. 91 (2003) 241804, [hep-ex/ 0307080 ].

[16] CDF, D0 Collaboration, A. V. Popov, Recent Heavy Flavors results from Tevatron, in Proceedings, 52nd Rencontres de Moriond on QCD and High Energy Interactions: La Thuile, Italy, March 25-April 1, 2017, pp. 71-74, 2017.

[17] PROSA Collaboration, M. V. Garzelli, S. Moch, O. Zenaiev, A. Cooper-Sarkar, A. Geiser, K. Lipka, R. Placakyte, and G. Sigl, Prompt neutrino fluxes in the atmosphere with PROSA parton distribution functions, JHEP 05 (2017) 004, [arXiv: 1611 . 03815].

[18] M. Benzke, M. V. Garzelli, B. Kniehl, G. Kramer, S. Moch, and G. Sigl, Prompt neutrinos from atmospheric charm in the general-mass variable-flavor-number scheme, JHEP 12 (2017) 021, [arXiv:1705.10386].

[19] ALICE Collaboration, S. Acharya et al., Measurement of D-meson production at mid-rapidity in pp collisions at $\sqrt{s}=7 \mathrm{TeV}$, Eur. Phys. J. C77 (2017), no. 8 550, [arXiv:1702.00766].

[20] Particle Data Group Collaboration, M. Tanabashi et al., Review of Particle Physics, Phys. Rev. D98 (2018), no. 3030001 .

[21] P. Marquard, A. V. Smirnov, V. A. Smirnov, M. Steinhauser, and D. Wellmann, $\overline{M S}$-on-shell quark mass relation up to four loops in QCD and a general $S U(N)$ gauge group, Phys. Rev. D94 (2016), no. 7 074025, [arXiv:1606.06754].

[22] LHCb Collaboration, R. Aaij et al., Measurement of B meson production cross-sections in proton-proton collisions at $\sqrt{s}=7 \mathrm{TeV}$, JHEP 08 (2013) 117, [arXiv:1306.3663]. 